\title{
DISPERSIÓN ASISTIDA POR MAGNETRÓN EN PELÍCULAS DE TiN
}

\author{
Rubin Ortega de la Rosa ${ }^{1}$ Claudia Franco M. ${ }^{2}$ Eduardo Valdés C. ${ }^{2}$ Guillermo de Anda Rodríguez
}

Recibido el 6 de octubre de 2004, aceptado el 18 de abril de 2005

\begin{abstract}
RESUMEN
Por medio de la técnica PVD (Deposición física en fase vapor), de dispersión asistida por un magnetrón, se depositó un recubrimiento de TiN sobre sustratos de acero grado herramienta D2 a partir de un blanco de titanio en una mezcla de gases de $\operatorname{Ar}$ y $\mathrm{N}_{2}$. Los recubrimientos fueron depositados a temperaturas alrededor de $400^{\circ} \mathrm{C}$ de sustrato, observándose una significativa variación en el color clásico dorado y la dureza. Los resultados obtenidos mediante SEM (Microscopio Electrónico de Barrido) y difracción de rayos X revelan que hubo un cambio de coloración, composición y dureza al variar las condiciones de tiempo de depositación y distancia de trabajo sobre los sustratos. Esto fue corroborado en los resultados obtenidos por mediciones de dureza con un microdurómetro Mitutoyo.

La microscopia electrónica de barrido reveló una morfología columnar. Utilizando un microindentador Vickers se obtuvo la dureza de los recubrimientos para las muestras analizadas.
\end{abstract}

Palabras clave: PVD, magnetrón, TiN, sustratos, SEM.

\section{ABSTRACT}

By means of the PVD (Physical Vapour Deposition) technique using magnetron assisted dispersion, a film of TiN it was deposited on substrates of tool grade steel D2, starting from a titanium target in a mixture of Ar and $N_{2}$ gases. The films was deposited at substrate temperatures around $400^{\circ} \mathrm{C}$. A significant surface change in the typical golden color and in the hardness was observed. The results obtained using SEM (Scanning Electron Microscope) and diffraction of X rays reveal that there was a change of coloration, composition and hardness when varying the conditions of deposition times and working distances. This was confirmed in the results obtained by hardness measurements using a Mitutoyo microdurometer. The scanning electronic microscopy revealed a columnar morphology. Vickers hardness measurements were performed on all samples.

Keywords: PVD, magnetron, TiN, substrates, SEM.

\section{INTRODUCCIÓN}

La evolución en el proceso de deposición física fase vapor (PVD) contribuye ampliamente a la aplicación de películas delgadas de alta dureza en herramientas de corte. En los últimos años el número de componentes a mecanizar ha aumentado de forma considerable, es por ello que los recubrimientos duros juegan un papel importante en la industria por mejorar la vida de la herramienta y para alcanzar propiedades que normalmente no son posibles de obtener en el sustrato por sí solo, como alta dureza, baja fricción, resistencia al desgaste, así como la alta resistencia a la corrosión y buena resistencia a altas temperaturas [1,2]. Las propiedades que pueden mejorarse se catalogan en seis categorías básicas: ópticas, eléctricas, magnéticas, químicas, mecánicas y térmicas [3]. Los recubrimientos de nitruro de titanio (TiN) además de las propiedades antes mencionadas buscan mejorar simultáneamente lo depositado sobre herramientas de corte, para incrementar substancialmente la dureza, baja el coeficiente de fricción [4] son conocidos por su brillante color oro y es utilizado también en aplicaciones decorativas [5]. La alta dureza, baja fricción y resistencia al desgaste de películas de TiN pueden ser directamente afectadas por los cambios en las condiciones de deposición [6,7]. Con respecto al claro colorido de películas delgadas, uno debe distinguir entre los coloridos inherentes (por ejemplo en nitruros, carbonitruros o boruros) y los coloridos claros, debido a la interferencia de los efectos (por ejemplo óxido transparente o extremadamente delgado de las películas absorbentes) [8]. Los coloridos claros de las películas

\footnotetext{
1 Instituto Tecnológico de Zacatecas, Carretera Panamericana entronque a Guadalajara s/n Apartado Postal 245, Tel/fax: (+52) 492 9245366, Zacatecas, México,rubin_ortega_2002@yahoo.com.mx

2 Instituto Tecnológico de Saltillo, Blvd. Venustiano Carranza 2400 Apdo. postal 600 c.p., Tel/fax (+52) 844 4389500, Saltillo, Coah., México

3 Instituto Tecnológico de San Luis Potosí, Av. Tecnológico s/n, c.p. 78437 tel (+ 52) 4448182136 ext 127 , San Luis Potosí, México.
} 
de la interferencia son influenciados principalmente por el espesor y la relación de gases $\mathrm{Ar}-\mathrm{N}_{2}$ [9]. Cuando se emplea nitrógeno como elemento de aportación como aleante o para la formación de compuestos, se produce un incremento de dureza superficial. Esto se debe a la formación de una capa de nitruro de titanio. Conforme se van cambiando las condiciones de distancia y tiempo de deposición, el color del recubrimiento cambiará, tendiendo a dorado, para un tiempo de $60 \mathrm{~min}$. Y distancia entre 54 y $61 \mathrm{~mm}$, por arriba o abajo de estas condiciones, el color variará significativamente, ya que la cinética de gases hará que se mezclen pequeñas cantidades de oxígeno, lo que causará oxidación, o también a colores combinados con dorado, de acuerdo a la estequiometría de la película.

\section{EXPERIMENTACIÓN}

\section{Depositación del recubrimiento}

El recubrimiento de TiN se depositó sobre un sustrato de acero grado herramienta D2, empleando un blanco de $\operatorname{Ti}(99.997 \%$ de pureza) a una temperatura de sustrato de $400{ }^{\circ} \mathrm{C}$, cuyas dimensiones son: $40 \mathrm{~mm} \times 16 \mathrm{~mm} \times 8 \mathrm{~mm}$. El equipo utilizado para realizar los recubrimientos fue un sistema de dispersión asistida por magnetrón vacuum modelo U.S. Este equipo alcanza un vacío residual de $5 \times 10^{6}$ Torr, empleando una bomba mecánica y una bomba secundaria difusora. Para mantener baja la temperatura del equipo se hace circular agua a través de él, lo que permite alcanzar una presión menor más rápidamente y que no exista un sobrecalentamiento que pudiera dañar el cátodo durante todo el proceso de deposición. Una vez logradas las condiciones máximas de vacío, se aplica una limpieza a la cámara en una atmósfera controlada de Ar y así eliminar la presencia de $\mathrm{O}_{2}$ que causaría oxidación de la película. Posteriormente se aplica una potencia de $77 \mathrm{~W}$ al cátodo. Una vez que se forma el plasma, se hace una limpieza de éste; con la muestra cubierta por medio de un obturador, después de 10 min. se descubre la muestra y se hace una limpieza al sustrato por dispersión, por 10 min., con la finalidad de formar una capa metálica de acoplamiento entre el sustrato y el recubrimiento y además eliminar imperfecciones superficiales y algunos contaminantes, al cabo de esta etapa, lo que mejora la adherencia del nitruro. Después se introduce $\mathrm{N}_{2}$ en la cámara iniciando la nitruración. Una vez que el $\mathrm{N}_{2}$ entra en la cámara, el color del plasma cambia de color pasando de morado a rosado, si hay exceso de nitrógeno, tomará un color rojizo. El tiempo de crecimiento del recubrimiento fue variando de 30 hasta $90 \mathrm{~min}$., variando la distancia blanco-sustrato.
Se realizaron 10 muestras llamadas M1, M2,.. M10, de acuerdo a las consideraciones anteriores de deposición distancia-tiempo. El depósito se obtuvo con un blanco de Ti de $5.0 \mathrm{~cm}$ de diámetro. Las muestras M1, M2 y M3 fueron depositadas en un tiempo de $30 \mathrm{~min}$., M4, M5, M6 y M7 en un tiempo de $60 \mathrm{~min}$. y M8, M9 y M10 en 90 min., variando también las condiciones de distancia según la tabla:

Tabla 1 Parámetros empleados en los recubrimientos sobre 10 muestras.

\begin{tabular}{|c|c|c|c|}
\hline $\mathrm{N}^{\mathbf{0}}$ & $\begin{array}{c}\text { Distancia } \\
(\mathrm{mm})\end{array}$ & $\begin{array}{c}\text { Temp. } \\
\left({ }^{\circ} \mathrm{C}\right)\end{array}$ & $\begin{array}{c}\text { Tiempo } \\
(\mathrm{min})\end{array}$ \\
\hline 1 & 61 & 423 & 30 \\
\hline 2 & 54 & 422 & 30 \\
\hline 3 & 47 & 429 & 30 \\
\hline 4 & 68 & 428 & 60 \\
\hline 5 & 61 & 425 & 60 \\
\hline 6 & 54 & 434 & 60 \\
\hline 7 & 47 & 430 & 60 \\
\hline 8 & 61 & 414 & 90 \\
\hline 9 & 54 & 421 & 90 \\
\hline 10 & 47 & 427 & 90 \\
\hline
\end{tabular}

Para estudiar la estructura del recubrimiento se utilizó un difractómetro de rayos X marca Phillips. La morfología de los recubrimientos, así como la medida de la dureza a partir de las huellas de indentación fueron realizadas con un microscopio electrónico de barrido modelo FEI modelo Quanta 2000 con EDX Oxford Instruments, trabajando en alto vacío y en los modos de electrones retrodispersados y secundarios. Este mismo sistema cuenta con una microsonda EDX y WDX que permite obtener la composición química de los recubrimientos.

\section{Análisis de dureza}

Se llevaron a cabo diez experimentos para obtener la dureza del recubrimiento por medio de un microdurómetro marca Mitutoyo y se analizaron las huellas en el microscopio electrónico de barrido.

\section{Análisis de color}

Las muestras obtenidas fueron depositadas con TiN, de acuerdo a la tabla anterior, observándose cambios de coloración. 


\section{RESULTADOS Y DISCUSIÓN}

\section{Análisis de color}

Tabla 2 Colorimetría en los recubrimientos sobre 10 muestras.

\begin{tabular}{|c|l|}
\hline MUESTRA & \multicolumn{1}{|c|}{ COLOR } \\
\hline 1 & Azul 90\%-dorado $10 \%$ \\
\hline 2 & Morado $70 \%$-dorado $30 \%$ \\
\hline 3 & Azul claro \\
\hline 4 & Azul 50\%-morado 50\% \\
\hline 5 & Dorado $100 \%$ \\
\hline 6 & Dorado 90\%-morado $10 \%$ \\
\hline 7 & Azul 30\%-dorado 50\%-morado $20 \%$ \\
\hline 8 & Verde 30\%-morado $70 \%$ \\
\hline 9 & Dorado $95 \%$-morado $5 \%$ \\
\hline 10 & Azul 50\%-dorado 30\%-morado $20 \%$ \\
\hline
\end{tabular}

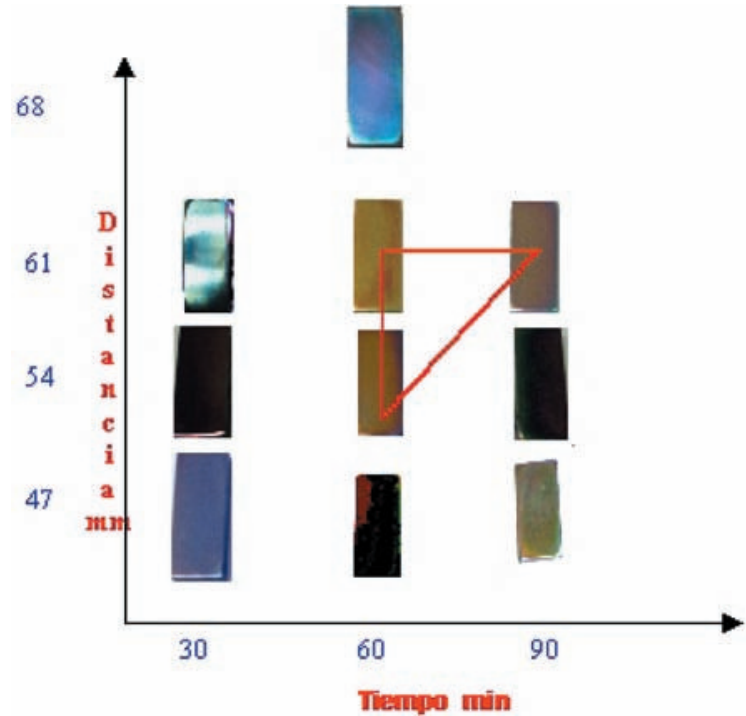

Fig. 1 Las muestras presentan diferentes coloraciones debido a la cinética de gases.

\section{Cinética de gases}

El plasma contiene una gran distribución de energía, la cual puede activar el proceso de deposición de películas a bajas temperaturas.

Las cuales pueden ser influenciadas por la presión de los gases, y distancia sustrato-blanco, de acuerdo a las moléculas de los gases que están continuamente colisionando entre sí al azar y con la superficie.

El intercambio de energía que tiene lugar durante estas colisiones conduce a una distribución en equilibrio de velocidades moleculares dada por la fórmula de Maxwell-Boltzmann, ecuación 1:

$$
\frac{d N / d c}{N}=4 \pi c^{2}\left(\frac{m}{2 \pi k_{B} T}\right)^{3 / 2} \exp \left(\frac{\frac{1}{2} m c^{2}}{k_{B} T}\right)
$$

Cuando un gas se calienta lo suficiente, los átomos colisionan entre sí y sacan a sus electrones, forman un plasma. El punto importante es que un gas ionizado tiene propiedades únicas. En la mayoría de los materiales la dinámica de movimiento está determinada por las fuerzas entre regiones vecinas del material. En el plasma, la separación de cargas entre iones y electrones aumenta los campos magnéticos. El comportamiento de vapores y gases, ambos en estado macroscópico y en escala molecular, es fundamental para entender la deposición de películas delgadas desde la fase vapor.

El flujo de impacto de moléculas sobre la superficie es fundamental para determinar la velocidad de depositación de la película. La ley de los gases ideales se aplica cuando el volumen de las moléculas en el gas es mucho más pequeño que el volumen del gas y cuando las fuerzas cohesivas entre las moléculas pueden ser despreciadas. Ambas de estas consideraciones son válidas cuando la concentración n es lo suficientemente baja, el espacio entre moléculas es mucho más grande que el diámetro molecular. Esto siempre se cumple en el caso de la deposición de películas delgadas desde la fase vapor, donde $\mathrm{T}$ está en o por encima de la temperatura ambiente y P está en o por debajo de $1 \mathrm{~atm}$. La ecuación de Knudsen es una de las más importantes relaciones en la tecnología de películas delgadas. Esto es válido en vacío y en regímenes de flujo operando (ecuación 2) una cierta presión.

$$
J_{i}\left(\frac{m c}{c m^{2} s e g}\right)=2.63 \times 10^{20} \frac{p}{\sqrt{M T}}
$$

Para obtener un valor de la magnitud del flujo de impacto, considere un elemento de masa atómica 40 , $25^{\circ} \mathrm{C}$ y $10^{-3} \mathrm{~Pa}, \mathrm{~J}_{\mathrm{i}}=2.4 \times 10^{15} \mathrm{~mol} / \mathrm{cm}^{2} \mathrm{seg}$. Si todo este gas que impacta es adsorbido y adherido por la superficie en el proceso de depositación de películas delgadas, ¿qué tan rápidamente se acumula? Para una molécula de diámetro $0.3 \mathrm{~nm}$, hay cerca de $10^{15}$ moléculas por capa molecular (monocapa), por lo que la velocidad de acumulación es de 2.4 monocapas $/ \mathrm{seg}$. o $2.6 \mu \mathrm{m} / \mathrm{hr}$, lo cual es una velocidad típica en los procesos de depositación de películas delgadas, aunque puede haber velocidades mucho más elevadas. Esto 
significa que solamente $10^{-8} \mathrm{~atm}$ de presión de gas reactante es necesaria para alcanzar esta velocidad de deposición si todo el gas que impacta sobre la superficie se está depositando. Esto también significa que si el gas tiene impurezas que están entrando a la cámara de depositación con una velocidad mayor que la del constituyente deseado en la película, la película tendrá un nivel alto de contaminación [9]. Para depositar una película $99.9 \%$ pura a $2.6 \mu \mathrm{m} / \mathrm{hr}$, la presión de las impurezas de los gases reactivos debe estar por debajo de $10^{-6} \mathrm{~Pa}$, lo cual es considerado que está dentro del rango de "ultra alto" vacío.

En algunos procesos de película delgada la velocidad de deposición es lineal, dh/dt, la cual es fácilmente determinada del grosor de la película, h, dividida por el tiempo de deposición. El flujo molecular de deposición (ecuación 3) puede ser encontrado de esta velocidad si la densidad de la película es conocida.

$J_{r}\left(\frac{m c}{\mathrm{~cm}^{2} \mathrm{seg}}\right)=1.67 \times 10^{16} \frac{\frac{d h}{d t}(\mu \mathrm{m} / \mathrm{h}) \rho_{m}\left(\mathrm{gr} / \mathrm{cm}^{3)}\right.}{M(\mathrm{gr} / \mathrm{mol})}$

donde $\mathrm{J}_{\mathrm{r}}$ es el flujo de deposición molecular y $\rho_{\mathrm{m}}$ es la densidad de la película. Es digno de recordar que para la mayoría de los sólidos la concentración molecular $\left(\rho_{\mathrm{m}} \mathrm{N}_{\mathrm{A}} / \mathrm{M}\right)$ es de el orden de $5 \times 10^{22} \mathrm{~mol} / \mathrm{cm}^{3}[10]$.

\section{Análisis de dureza}

Se llevaron a cabo diez experimentos para obtener la dureza del recubrimiento, por medio de un microdurómetro marca Mitutoyo y se analizaron las huellas en el microscopio electrónico de barrido.

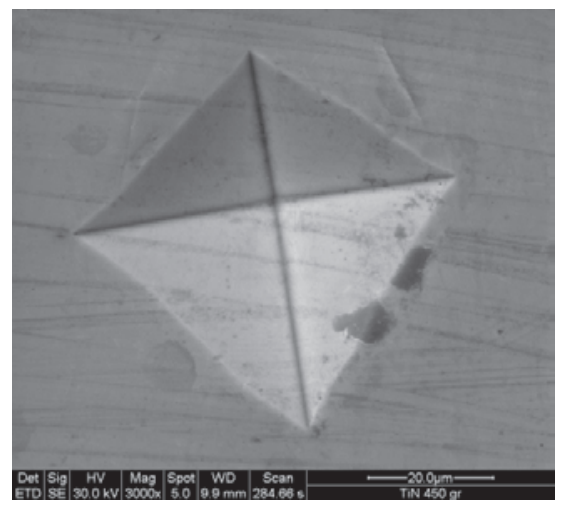

Fig. 2 Huella de indentación Vickers, a $450 \mathrm{~g}$ realizada sobre un recubrimiento de TiN con un espesor de $3 \mu$, con un tiempo de deposición de 60 min. Para las muestras M5.

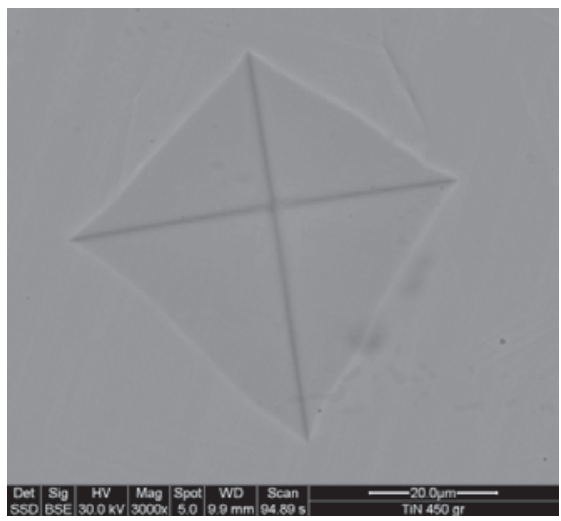

Fig. 3 Huella de indentación Vickers a $450 \mathrm{~g}$ realizada sobre un recubrimiento de TiN con un espesor de $3 \mu$, con un tiempo de deposición de $60 \mathrm{~min}$. Para la muestra M6.

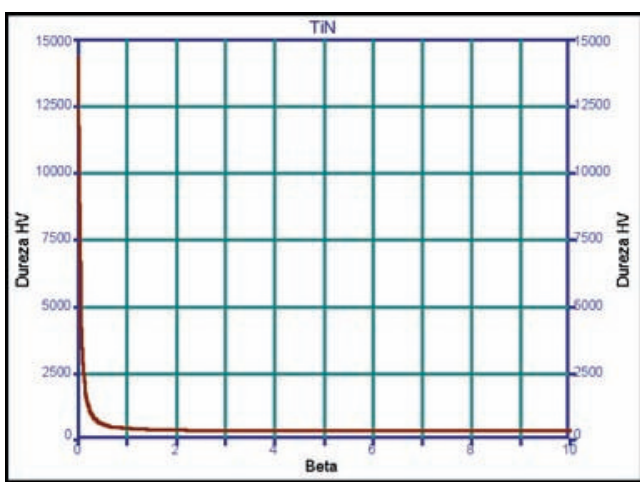

Fig. 4 Gráfica lineal del ajuste de datos para obtener la microdureza del TiN para las muestras M5 y M6.

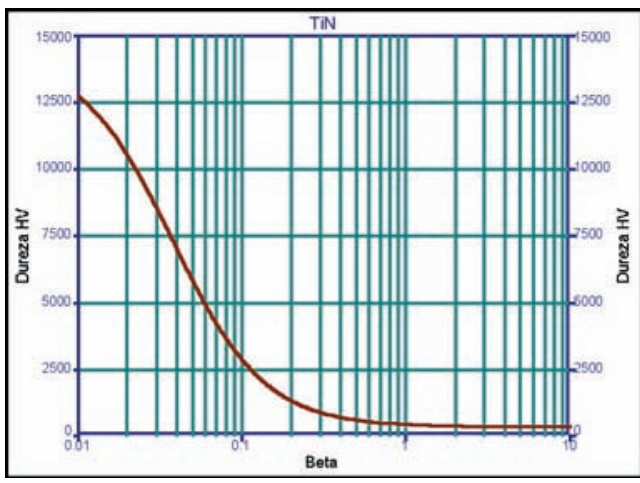

Fig. 5 Gráfica logarítmica del ajuste de datos para obtener la microdureza del TiN para las muestras M5 y M6.

La gráfica obtenida con los valores medidos y con los datos de ajuste se muestra en las Figuras 4 y 5 . Con este ajuste se obtienen los siguientes valores de dureza: para el sustrato 3.59 Gpa y para la película $39.22 \mathrm{Gpa}$. 
Esta dureza está por encima de lo reportado en la literatura; sin embargo, en el manejo de esta técnica se observó que existe una gran sensibilidad en el manejo de los datos, dio buenos resultados que se espera se puedan mejorar con el incremento y logro de mediciones menores a los $15 \mathrm{~g}$.

\section{Caracterización}

Las muestras fueron cortadas para ser observadas y analizadas en microscopia electrónica de barrido, EDX, WDX. Además se les midió microdureza Vickers.

En microscopia electrónica de barrido la muestra se analizó por la cara superior y transversalmente para medir su espesor y hacerle un análisis de composición por EDX y WDX.

Inicialmente, se analizó el recubrimiento por la parte superior, y se corroboró TiN, verificándose esto con mapeo en línea por EDX y después puntual con WDX. Se realizó mapeo en línea con la finalidad de comprobar que el recubrimiento era más grueso en las orillas que en el centro del sustrato, obteniéndose el espectro que se muestra en la Figura 6. El análisis por WDX se hizo para comprobar que se tenía nitrógeno en el recubrimiento, ya que el EDX tiene una menor capacidad para detectar elementos ligeros, aunque con el EDX con el que cuenta el SEM de la institución se puede detectar a partir del berilio.

\section{TiN}

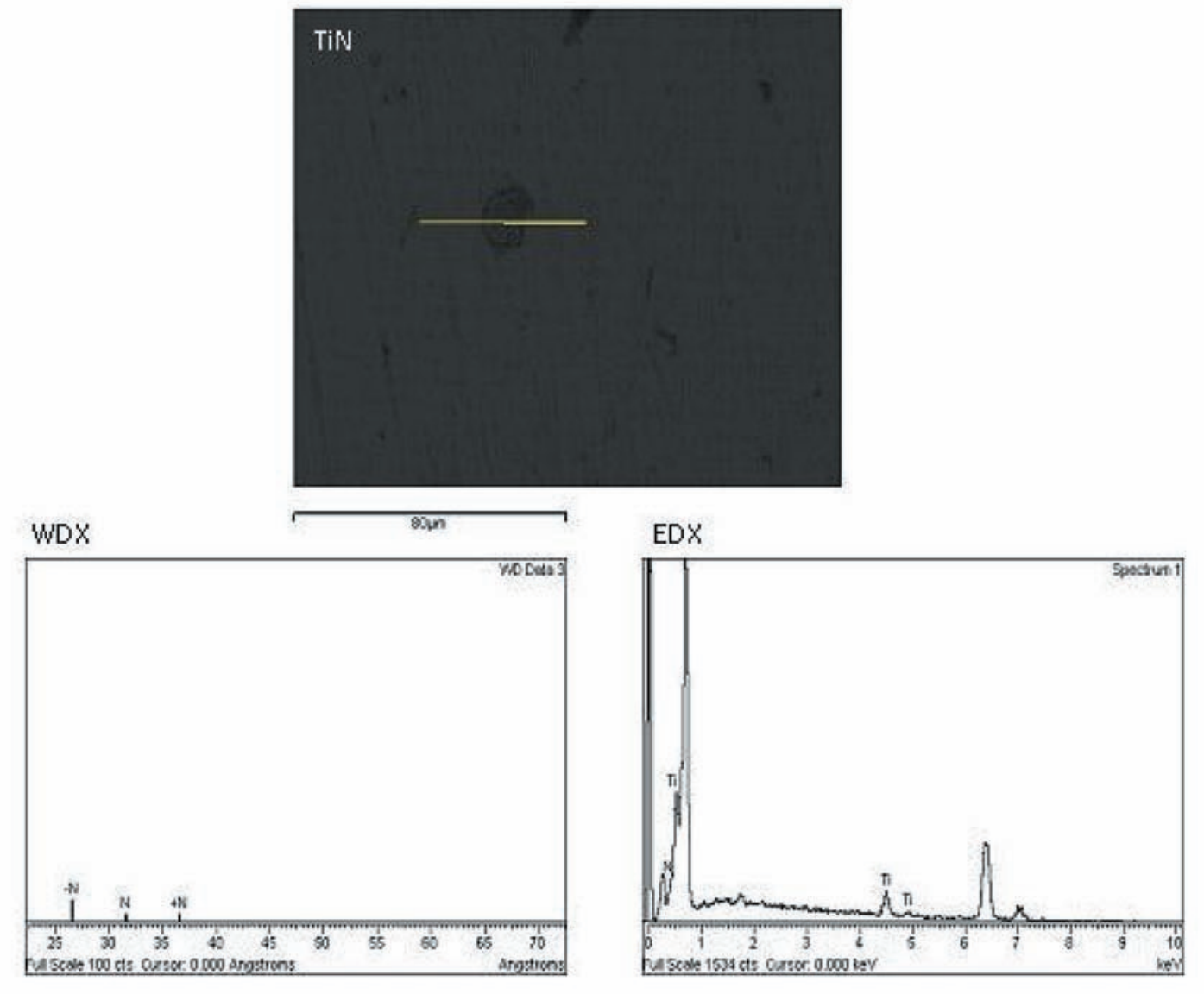

Fig. 6 Micrografía de la superficie del recubrimiento y barrido lineal composicional para confirmar la existencia del TiN en el recubrimiento. 
Se prepararon muestras transversales con el fin de observar el espesor de la película; sin embargo, en un análisis composicional lineal realizado por EDX se pueden apreciar picos de $\mathrm{Ti}$ y $\mathrm{N}_{2}$, por lo que esto sirve de referencia del espesor del recubrimiento en el sustrato sometido a $60 \mathrm{~min}$. de deposición, siendo este espesor

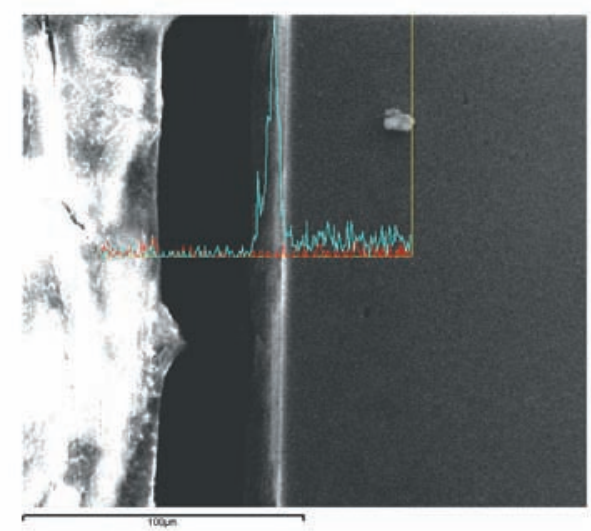

de $3 \mu \mathrm{m}$. El espesor fue medido con ayuda de un software de análisis de imágenes XT Docu V. 3.2 implementado en el SEM, tomando en cuenta sólo la parte más brillante de la imagen, siendo esta la cual se acerca al pequeño pico de $\mathrm{N}_{2}$ que aparece en el espectro. Las imágenes se muestran en la Figura 7.

Fig. 7 Análisis composicional lineal por EDX de un recubrimiento de TiN.
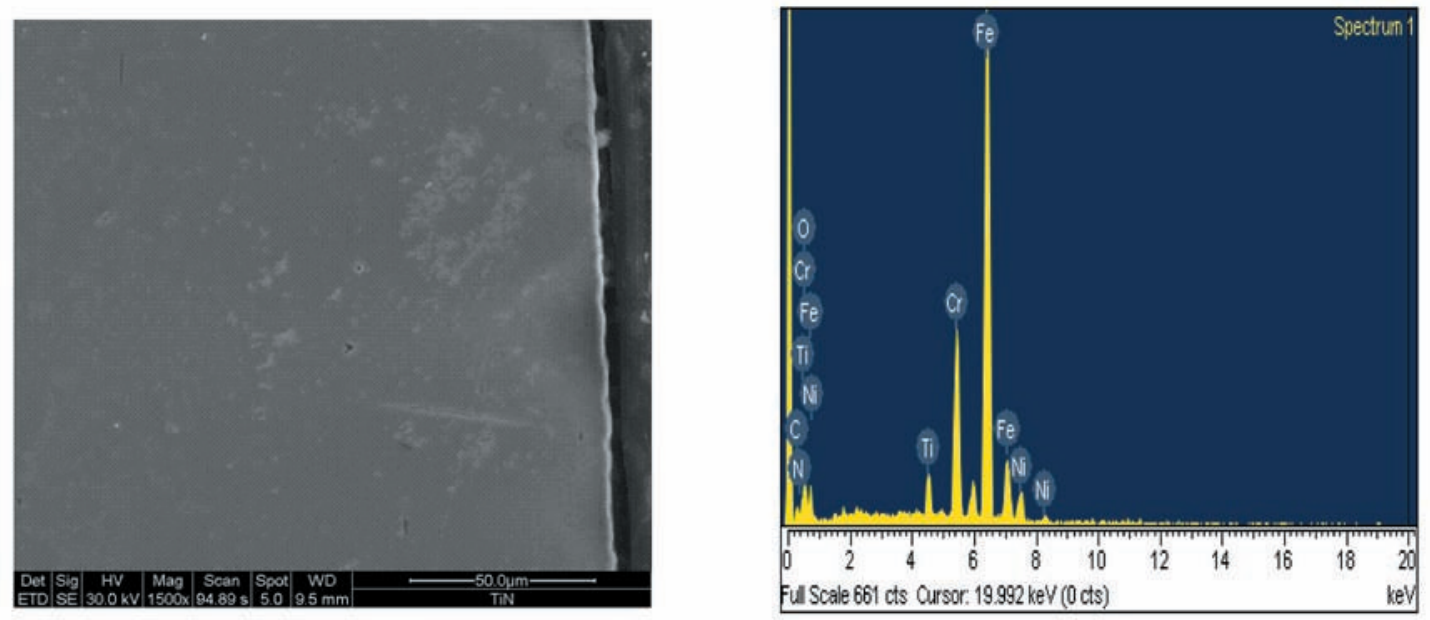

Fig. 8 Micrografía y espectro de energía para un acero recubierto con una película de TiN. Muestra M5. 


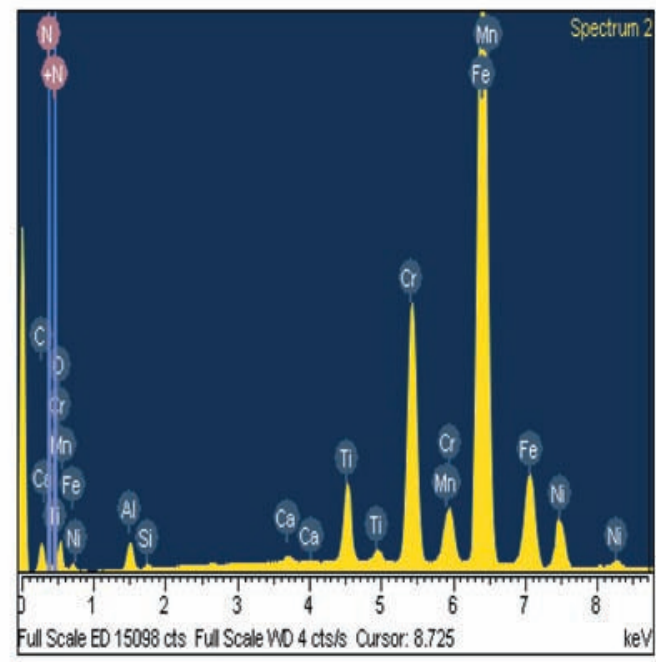

Fig. 9 Micrografía y espectro de energía y longitud de onda para un acero recubierto con una película de TiN depositada a $400{ }^{\circ} \mathrm{C}$. Muestra M6.
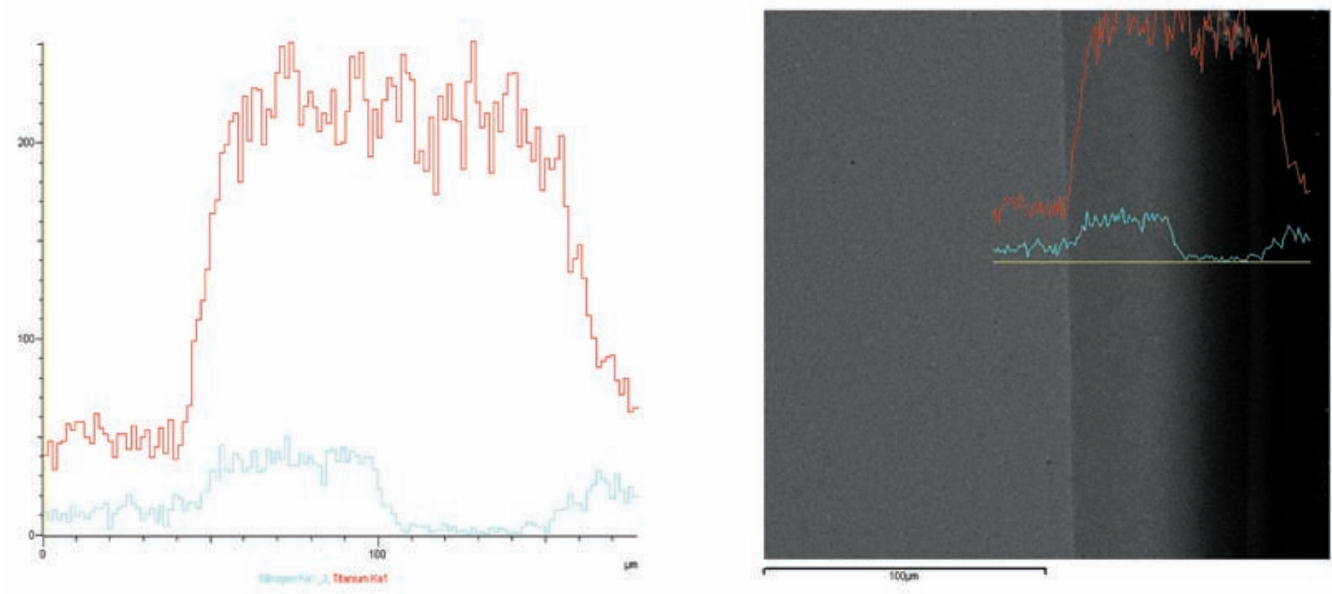

Fig. 10 Microanálisis lineal en películas de TiN depositadas por 60 min. a muestras M5 y M6 a $400^{\circ} \mathrm{C}$.

\section{CONCLUSIONES}

Se estudió el efecto de la distancia del blanco al sustrato sobre las propiedades mecánicas y estructurales de los recubrimientos de TiN fabricados por dispersión asistida por un magnetrón (Sputtering).

El incremento de la distancia impide la formación de la fase TiN y por lo tanto cambia su color característico dorado y considerablemente la dureza de los recubrimientos. Cuando se llega a la distancia adecuada los recubrimientos mejoran la dureza de la película, pues se forma un material estable y con el efecto de la temperatura del sustrato se presenta una mejor adhesión.
A mayor temperatura mayor dureza y menor espesor en el recubrimiento. Se estimó el valor de la dureza real de los recubrimientos, eliminando el efecto del sustrato, aplicando el modelo de dureza basado en el trabajo de indentación.

\section{AGRADECIMIENTOS}

Agradecemos al COSNET (Consejo del Sistema Nacional de Educación Tecnológica), México, su apoyo para el financiamiento de este trabajo, al CONACYT (Consejo Nacional de Ciencia y Tecnología), México, financiamiento del proyecto. A los Drs. José M. Albella del ICMM (Instituto de Ciencia de Materiales de Madrid) España, Luis Nerey Luna de la Universidad de los Andes, sus valiosas sugerencias. 


\section{REFERENCIAS}

[1] L. Cunha, M. Andritschky, L. Rebouta and K. Pisehow. "Corrosion of CrN and (TiAl)N Coatings in Chloride-Containing Atmospheres". Surface and Coatings Technology, no. 116: 1152-1160. 1999.

[2] Da-Yung Wang, Yen-Way Li, Chi-Long Chang and Wei-Yu Ho. "Deposition of high quality (Ti,Al)N hard coating by vacuum arc evaporation process". Surface and Coatings Technology, no. 114: 109113. 1999.

[3] D.L. Smith. "Thin-Film Deposition", Mc Graw Hill. 1995.

[4] T.A. Li, H. Li, F. Pan. "Microstructure and nanoindentation hardness of Ti/TiN multilayered films". Surface and Coatings Technology, 137: 225229. 2001.
[5] E. Harju, S. Kivivuori and A.S. Korhonen, Surf. Coat. Technol. 98, 112. 1999.

[6] A. Matthews and A. R. Lefkow. "Problems in the physical vapour deposition of titanium nitride", Thin Solid Films, 126: 283-291. 1985.

[7] R. Manory. "Effects of deposition parameters on structure and composition of reactively sputtered TiN $_{X}$ films”, Surface Engineering, 3: 233-240. 1987.

[8] R. Franchy. "Growth of thin, crystalline oxide, nitride and oxynitride films on metal and metal alloy surfaces", Surface Science Reports, no. 38: 195294, 2000.

[9] M. Ohring. "The Materials Science of Thin Films", Academic Press Inc, San Diego. 1992.

[10] C.W. Kimbiln, Journal of Applied Physics, 45, 5235. 1974. 